\title{
INFLUÊNCIA DO ETANOL DAS BEBIDAS ALCOÓLICAS NA ATEROSCLEROSE EM ARTÉRIAS CARÓTIDAS EXTRACRANIANAS
}

\author{
Ibsen Thadeo Damiani ${ }^{1}$, Rubens J osé Gagliardi², Milberto Scaff ${ }^{3}$
}

\begin{abstract}
RESUMO - Existem fortesevidências de menor incidência de doença cerebrovascular odusiva, de aterosclerose coronariana e de outros vasos em indivíduos com consumo leve ou moderado de álcool. Este estudo procura analisar o efeito do etanol, em diferentes dosesno comportamento da aterosclerose carotídea extracraniana. Através do ultrassom Doppler colorido, foram investigadas 328 artérias carótidas extracranianas, de homens e mulheres brancos, com mais de 35 anos de idade, normotensos, não tabagistase sem as principais doenças que constituam fatores de risco para doenças cardiovasculares. Foram divididos de acordo com o consumo de álcool por semana (em mililitros) em abstêmios, etilistas leves (1 a 100), moderados (101 a 300) e pesados (301 ou mais). Houve menor incidência de placas de aterosclerose e de estenose naqueles que ingeriram moderada quantidade. Conclusão: O estudo sugere uma ação protetora do álcool etílico para aterosclerose carotídea, quando ingerido em moderada quantidade.
\end{abstract}

PALAVRAS-CHAVE: álcool, carótidas, aterosclerose.

\begin{abstract}
The influence of the ethanol in alcoholic beverages in the extracranial carotid arteries atherosclerosis
ABSTRACT - There is less incidence of occlusive cerebrovascular disease, of coronarian atherosclerosis and of other arteries with a light to moderate consumption of alcohol, suggesting that the same may occur with respect with the extracranial carotid arteries. Using color Doppler ultrasonography, we studied 328 extracranial carotid arteries of white male and females over 35 year old, with normal blood pressure, non-smokers and free of the main diseases make up the risk factors for cardiovascular diseases. They were stratified, according to the level of weekly alcohol consumption in milliliters ( $\mathrm{ml}$ ), in abstainers, light drinkers (1 to 100), moderate drinkers (101 to 300) and heavy drinkers (over 301). There was a lower incidence of atherosclerotic plaque and stenosis in the moderate drinkers. Conclusion: The study suggests that ethyl alcohol when drunk with moderation exerts a protective action from carotid atherosclerosis.
\end{abstract}

KEY WORDS: alcohol, carotid, atherosclerosis.

Há anos, a relação entre álcool e doença cerebrovascular (DCV) tem sido firmemente estabelecida, seja na forma oclusiva ou hemorrágica ${ }^{1-3}$. O efeito do álcool etílico sobre a aterosclerose, em especial das artérias carótidas extracranianas, é motivo de controvérsias, havendo poucos estudosque examinaram esta relação e seu mecanismo ${ }^{4-6}$. Décadas de pesquisas epidemiológicas têm demonstrado que a aterosderose tem uma etiologia multifatorial, dificultando a análise quantitativa e qualitativa de um fator de risco isoladamente, osquaisinteragem entre $\mathrm{si}^{7-10}$.

Parece existir uma associação inversa significante entre baixo consumo de álcool e aterosclerose carotídea $11,-15$, a qual é demonstrada em forma de curva tipo "U"6,16 ou "J"17. Estes dados poderiam explicar o fato de que o baixo ou o moderado consumo de álcool tem ação protetora contra DCV odusiva ${ }^{18}$. Porém, existem autores que não encontram esta associação ${ }^{19}$.

O objetivo deste estudo é avaliar, através de exame de ultrassonografia colorida com Doppler, a presença de placas de ateroma e o grau de estenose em artérias carótidas extracranianas em abstêmios e etilistas com diferentes graus de consumo alcoólico. Diferentemente dostrabalhoscitadosna revisão acima, somente o consumo alcoólico deverá ser o fator de risco existente; doentes com outrosfatores

Disciplina de Neurologia da Faculdade de Medicina da Santa Casa de São Paulo (FMSCSP), São Paulo SP, Brasil; Divisão de Clínica Neurológica do Hospital das Clínicas da Faculdade de Medicina da Universidade de São Paulo (HC- FMUSP), São Paulo SP, Brasil: 1Professor Instrutor da FM SCSP, ${ }^{2}$ Professor Adjunto da FMSCSP, ${ }^{3}$ Professor Titular de Neurologia da FMUSP.

Recebido 13 Fevereiro 2004, recebido na forma final 7 Maio 2004. Aceito 7 Julho 2004.

Dr. Ibsen Thadeo Damiani - Rua Espártaco 109 - 05045-000 São Paulo SP - Brasil. 
de risco clássicos para a aterosclerose foram excluídos, para permitir uma análise isolada da possível ação do álcool.

\section{MÉTODO}

Estudo prospectivo com pacientes que foram avaliadose acompanhados no Ambulatório de Neurologia da Santa Casa de São Paulo e em clínica particular, no período de 1990 a 1998.

Foram incluídos doentes de ambos os sexos, com idade acima de 35 anos e da raça branca. Optou-se por incluir apenas brancos por constituírem a maior parte da amostra inicial e devido às possíveis diferençasentre incidência de aterosclerose intra-extracraniana nas diferentes raças, aumentando as variáveise dificultando as análises e o objetivo deste estudo.

Os critérios de exclusão foram: tabagismo, exceto o fumante passivo; hipertensão arterial (PA > $140 \times 90$ $\mathrm{mmHg}$ ); obesidade, avaliada pelo índice de massa corpórea ( $\left(=\right.$ peso/altura ${ }^{2}$, menor que 25$)$; diabetes mellitus; dislipidemia e hiperuricemia (relatadaspelo paciente ou detectadas por exame prévio); uso de anticoagulante ou antiagregante plaquetário, de bloqueador de canal de cálcio, uso de anticoncepcional hormonal; doença autoimune ou em uso de imunossupressor.

O grupo controle (abstêmios) foi constituído de pessoas com cefaléia, doença infecciosa, distúrbios psicológicoscontroladosou lesão traumática sem conseqüências.

Os pacientes foram informados dos objetivos deste estudo, assinaram termo de consentimento informado e o estudo foi feito baseado em protocolo aprovado pelo Comitê de Ética da Santa Casa de São Paulo.

A média de consumo alcoólico foi obtida através de um questionário padrão, sendo feita a conversão das bebidas alcoólicas pelo teor de álcool etílico, quantificado em mililitros por semana e classificados, conforme o modelo de Handa et al., em abstêmios, alcoólicos leves (1 a $100 \mathrm{ml}$ ), moderados (101 a 200), e pesados (mais de $300 \mathrm{ml})^{4}$.

Para a análise estatística dos dados, foi utilizada a primeira edição em português do programa Epi-Info, versão 6, um sistema de processamento de texto, banco de dadose estatística para epidemiologia em microcomputadoresIBM-compatíveis. Os resultadosestatísticos foram avaliadospelo teste do Qui-quadrado ou teste Exato de Fisher, e análise da variança. Adotou-se o nível de confiança de 5\% para a decisão estatística.

\section{RESULTADOS}

Foram examinados 164 indivíduos no período de 1990 a 1998, dosquais 109 são abstêmios. A distribuição conforme o sexo e o grau de etilismo está demonstrada na Tabela 1.

Asidades variaram de 35 a 89 anos, sendo a média da amostra de 62,0 $\pm 14,0$ anos. Pela análise da variação dasidadesmédias dos diferentes graus de etilismos, nos homens, não houve diferença significativa entre asmesmas $(F=0,97$ e $p>0,10)$. O mesmo ocorreu entre as mulheres ( $F=0,97$ e $p>0,07)$.

Não houve diferença significativa entre os valores médios de colesterol total, LDL, glicemia de jejum, índice de massa corporal, plaquetas, níveis de transaminases, fibrinogênio e gama-GT para ambos os sexos.

Houve diferença significativa entre a média dos valores de HDL das etilistas pesadas com os demais graus de etilismo. Houve diferença significativa dasmédias de triglicérides entre abstêmiose os demais graus de etilismo, sugerindo aumento dos valores como alcoolismo; não houve, no entanto, diferença significativa entre as médias dos etilistas leves, moderados e pesados. Nas

Tabela1. Distribuição dos indivíduos de acordo com o grau de etilismo e sexo, de 1990/1998.

\begin{tabular}{lc}
\hline Grau de etilismo & Número (\%) \\
\hline Abstêmios (Grupo Controle) & 109 \\
Homens & $27(24,8)$ \\
Mulheres & $82(75,2)$ \\
Etilistas leves (1 a $100 \mathrm{ml}$ de álcool por semana) & 23 \\
Homens & $8(34,8)$ \\
Mulheres & $15(65,2)$ \\
Etilistas moderados (101 a $300 \mathrm{ml}$ de álcool por semana) & 12 \\
Homens & $8(66,7)$ \\
Mulheres & $4(33,3)$ \\
Etilistas pesados (mais de $300 \mathrm{ml}$ de álcool por semana) & 20 \\
Homens & $17(85,0)$ \\
Mulheres & $3(15,0)$ \\
Total & 164 \\
\hline
\end{tabular}


Tabela 2. Incidência de placas de aterosclerose nas artérias carótidas extracranianas em pessoas, de acordo com o grau de etilismo e sexo, de 1990/1998.

\begin{tabular}{llcc}
\hline Grau de etilismo & \multicolumn{2}{l}{ Pessoas com placas } & Total \\
& $\mathrm{n}$ & $\%$ & \\
\hline Abstêmios & & & \\
$\quad$ Homens & 17 & $17(63,0)$ & 27 \\
$\quad$ Mulheres & 44 & $(53,7)$ & 82 \\
Etilistas leves & & & \\
$\quad$ Homens & 3 & $(37,5)$ & 8 \\
$\quad$ Mulheres & 7 & $(46,6)$ & 15 \\
Etilistas moderados & & & \\
$\quad$ Homens & 2 & $(25,0)$ & 8 \\
$\quad$ Mulheres & 3 & $(75,0)$ & 4 \\
Etilistas pesados & & & \\
$\quad$ Homens & 7 & $(41,2)$ & 17 \\
$\quad$ Mulheres & 1 & $(33,3)$ & 3 \\
Total & 81 & $(49,4)$ & 164 \\
\hline
\end{tabular}

Tabela 3. Incidência de placas de aterosclerose em ambas as artérias carótidas extracranianas de cada indivíduo, de acordo com o grau de etilismo e sexo.

\begin{tabular}{llcc}
\hline Grau de etilismo & \multicolumn{2}{l}{ Carótidas com placas } & Total \\
& $n$ & $\%$ & \\
\hline Abstêmios & & & \\
$\quad$ Homens & 30 & $(55,5)$ & 54 \\
$\quad$ Mulheres & 76 & $(46,3)$ & 164 \\
$\begin{array}{l}\text { Etilistas leves } \\
\text { Homens }\end{array}$ & & & \\
$\quad$ Mulheres & 11 & $(37,5)$ & 16 \\
$\begin{array}{l}\text { Etilistas moderados } \\
\text { Homens }\end{array}$ & & $(36,7)$ & 30 \\
$\quad$ Mulheres & 2 & & \\
Etilistas pesados & 5 & $(12,5)$ & 14 \\
$\quad$ Homens & & $(62,5)$ & 8 \\
$\quad$ Mulheres & 11 & $(32,3)$ & 34 \\
Total & 2 & $(33,3)$ & 6 \\
\hline
\end{tabular}

Tabela 4. Risco relativo (RR) de placas de aterosclerose nas artérias carótidas de cada indivíduo, de acordo com o grau de etilismo e sexo (intervalo de confiança de 95\%).

\begin{tabular}{lc}
\hline Grau de etilismo & RR (intervalo de confiança de 95\%) \\
\hline Etilistas leves & \\
Homens & $0,68(0,34$ a 1,33$)$ \\
Mulheres & $0,79(0,48$ a 1,30$)$ \\
Etilistas moderados & \\
Homens & $0,26(0,07$ a 0,95$)$ \\
Mulheres & $1,35(0,77$ a 2,36$)$ \\
Etilistas pesados & \\
Homens & $0,58(0,34$ a 1,00$)$ \\
Mulheres & $0,72(0,23$ a 2,26$)$ \\
\hline
\end{tabular}

mulheres, houve diferença significativa entre as médias dos valores de triglicérides das etilistas moderadas e os demais graus de etilismo. Não se observou relação inversa e linear entre o consumo de etanol e agregação plaquetária, apesar de ter sido observado hipoagregação entre as etilistas pesadas. Houve diferença significativa entre osvalores de ácido úrico de abstêmios e etilistas pesa$\operatorname{dos}(p<0,05)$.

A Tabela 2 mostra a incidência de pessoas com placas de aterosclerose nas artérias carótidas extracranianas, de acordo com o grau de etilismo e sexo. Os testes estatísticos não demostraram diferença significativa entre estes valores para ambosossexos separadamente.

Osdemais resultados podem ser vistosna Figura 1 e nas Tabelas 3,4 e 5 .

Não foi possível comparar os diferentestipos de bebidas alcoólicas quanto à incidência de aterosclerose carotídea, porque a casuística foi pequena.

\section{DISCUSSÃO}

Oscritérios de inclusão e exclusão dosindivíduos dificultaram a obtenção de amostra maior, com pessoas com algum grau de etilismo e que não tenham outro fator de risco para aterosclerose. Há controvérsias se o consumo de álcool aumenta ou diminui osníveis de pressão arterial, e para eliminálasoptamos por trabalhar com normotensos, o que gera grande diferença entre esta pesquisa e os demais trabalhos já publicados ${ }^{4,6,7,15,-27}$.

Apesar de não haver diferença significativa, os dados da Tabela 2 sugerem, nos homens, uma diminuição da incidência de placas no etilismo até consumo moderado, tendendo a um aumento no etilismo pesado $(p>0,1)$. Se modificarmos a dis-

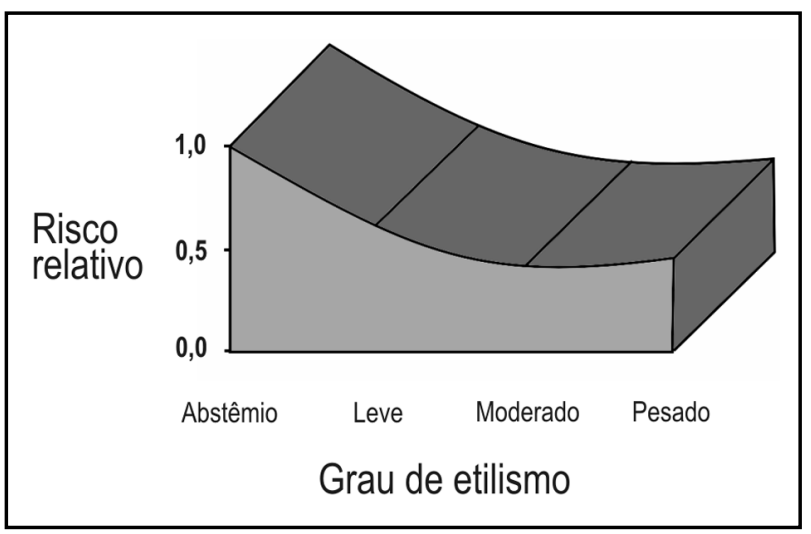

Fig 1. Risco relativo de placas de aterosclerose nas artérias carótidas extracranianas em homens de acordo com o grau de consumo alcoólico. 
Tabela 5. Riscos relativose respectivos intervalos de confiança (95\%), quanto à presença e o grau de estenose nas artérias carótidas extracranianas, em indivíduos etilistas com relação aos abstêmios.

\begin{tabular}{|c|c|c|c|c|}
\hline Grau de etilismo & Sem estenose & Estenose de até $50 \%$ & Estenose de 50 a $75 \%$ & Estenose $>75 \%$ \\
\hline \multicolumn{5}{|l|}{ Etilistas leves } \\
\hline Homens & $1,13(0,63$ a 2,00$)$ & $1,13(0,63$ a 2,00$)$ & -- (--) & -- (--) \\
\hline Mulheres & $1,38(1,01 \text { a } 1,91)^{*}$ & $0,86(0,52$ a 1,42$)$ & $--(--)$ & $--(--)$ \\
\hline \multicolumn{5}{|c|}{ Etilistas moderados } \\
\hline Homens & $1,69(1,12 \text { a } 2,55)^{*}$ & $0,56(0,23$ a 1,38$)$ & $--(--)$ & -- (--) \\
\hline Mulheres & $0,82(0,33$ a 2,04$)$ & $1,46(0,83$ a 2,58$)$ & $--(--)$ & $--(--)$ \\
\hline \multicolumn{5}{|l|}{ Etilistas pesados } \\
\hline Homens & $1,39(0,93$ a 2,07$)$ & $0,86(0,51$ a 1,45$)$ & $--(--)$ & $--(--)$ \\
\hline Mulheres & $1,46(0,81$ a 2,63$)$ & $0,78(0,25$ a 2,46$)$ & $--(--)$ & $--(--)$ \\
\hline
\end{tabular}

*significativo a $5 \%$

tribuição dospacientes e considerarmososetilistas (homens) levese moderadoscomo sendo um único grupo, aumentando o tamanho da amostra, a diferença se torna significativa $(p<0,05)$. $O$ valor do risco relativo foi menor para homens etilistas moderados $(0,4)$, embora as diferenças com os valores das demais categorias não tenham sido significativas. Num gráfico, estes dados caracterizaram uma curva em forma de "U" (Fig 1). Com relação às mulheres, a análise ficou prejudicada porque a amostra foi pequena.

Bogousslavsky et al. encontraram um risco relativo de 0,38 para aterosclerose carotídea por consumo leve - moderado de álcool $(\leq 354 \mathrm{ml} / \mathrm{sem}$. ou $\leq 50 \mathrm{ml} / \mathrm{d}$.), comparado com nenhum consumo, concluindo haver relação inversa e linear. Neste caso os etilistas pesadoseram muito poucos, não sendo possível conclusão12. Kiechl et al. descrevem uma associação entre álcool e aterosclerose carotídea tipo curva em " $U$ ", sendo o consumo ideal de etanol entre 1,3 a 63,3 $\mathrm{ml}$ por dia 6 .

No presente estudo, o risco relativo doshomens etilistas de não apresentarem estenose foi maior do que nos abstêmios, com uma associação significativa com o consumo moderado de álcool. NasmuIheres, o risco relativo de não apresentarem estenose foi maior e significativo nas etilistas leves. Com relação à presença de estenose de até $50 \%$ nos etilistas, não houve variação significativa. Pelo teste exato de Fisher, a não ocorrência de estenose entre 50 e $75 \%$ ou maior que $75 \%$ para os etilistas leves, moderadose pesados de ambos os sexos pode ser atribuída ao acaso.

Alguns fatores de risco apresentaram dados cujas diferenças eram significativas entre algumas categorias, como por exemplo, o HDL colesterol, aumentado nasmulheres etilistas pesadas somente. O mesmo ocorreu com os triglicérides, agregação plaquetária e ácido úrico. Estasalteraçõesnão podem ser atribuídas ao uso de bebida alcoólica, devendo ter ocorrido ao acaso, por não haver consistência clínica.

Como se verificou que a ação do álcool na aterogênese não depende dos demais fatores de risco para doenças cerebrovasculares, pode-se sugerir que um possível mecanismo de proteção é através do retardo ou não desenvolvimento de aterosclerose carotídea, por efeito direto sobre o endotélio, estando de acordo com estudo de Demirovic et al. ${ }^{5}$.

As eventuais diferenças nos resultados dostrabalhosé previsível, pois os dados sobre o consumo alcoólico variam entre eles. A investigação sobre os hábitos de cada indivíduo pesquisado é baseada em questionários, sendo freqüentemente incompleta, mesmo em serviçosuniversitários, onde consta do roteiro da anamnese ${ }^{28}$. Muitas vezes, os participantes eram voluntários, podendo causar viés, já que alcoólatrase etilistaspesados não o são ${ }^{23}$. Questionáriossobre o volume de álcool ingerido emestudos epidemiológicostêm sido criticados porque pode haver subestimativa, especialmente entre etilistas pesados ${ }^{5}$.

Existe diferença na composição alcoólica de uma mesma bebida entre diferentes regiões de estudo. As bebidas alcoólicas apresentam outros componentes, como por exemplo os flavonóides, os quais são pigmentos encontrados em vegetais com muitas ações bioquímicas, como antioxidantes, inibição da peroxidação lipídica e hipoagregação plaquetária ${ }^{29}$. Isto dificulta ainda maisa comparação entre diferentes trabalhos.

Considerando-se o exposto, as pessoas que consumiram bebidas alcoólicas de forma moderada (100 a 300 ml de álcool etílico por semana) apresentaram risco relativo para desenvolver aterosclerose carotídea de 0,4 com relação aos abstêmios, suge- 
rindo uma ação protetora do álcool, independente do tipo ou qualidade da bebida. Houve associação significante entre o consumo de álcool e a severidade da aterosderose carotídea, tipo curva em " $U$ ", revelando tendência em aumentar a incidência de aterosclerose carotídea com níveis elevados. Independente desta relação, o etilismo pesado está associado com dano a múltiplosórgãos sistêmicos e não pode ser recomendado. A evidência de que o consumo leve-moderado de álcool tem efeito protetor, contudo, pode ter implicações clínicas, quando corretamente indicado por um profissional médico.

\section{REFERÊNCIAS}

1. Hillbom $\mathrm{M}$. What supports the role of alcohol as a risk factor for stroke? Acta Med Scand Suppl, 1987;717:93-106.

2. Hillbom M, Kaste M. Alcohol abuse and brain infaction. Ann Med,1990;22:347-352.

3. Zuber M, Mas JL. Epidemiology of cerebrovascular accidents. Rev Neurol (Paris) 1992;148:243-255.

4. Handa K, Sasaki J, Saku K, Kono S, Arakawa K. Alcohol consumption, serum lipids and severity of angiographically determined coronary artery disease. Am J Cardiol, 1990;65:287-289.

5. Demirovic J, Nabulsi A, Folsom AR, et al. Alcohol consumption and ultrasonographically assessed carotid artery wall thickness and distensibility. The Atherosclerosis Risk in Communities (ARIC) Study Investigators. Circulation 1993;88:2787-2793.

6. Kiechl S, Willeit J, Egger G, Oberhollenzer M, Aichner F. Alcohol consumption and carotid atherosclerosis: evidence of dose-dependent atherogenic and antiatherogenic effects. Results from the Bruneck study. Stroke,1994;25:1593-1598.

7. Criqui MH. Epidemiology of atherosclerosis: an updated overview. Am J Cardiol, 1986;57:18C-23C.

8. Moore RD, Pearson TA. Moderate alcohol consumption and coronary artery disease: a review. Medicine (Baltimore) 1986;65:242-267.

9. Tell GS, Howard G, McKinney WM. Risk factors for site specific extracranial carotid artery plaque distribuition as measured by B-mode ultrasound. J Clin Epidemiol, 1989;42:551-559.

10. Connor EB. Obesity, hypertension and stroke. Clin Exper Hypertens, 1990;12:769-782.

11. Carolei A, Marini C, Ferranti E, Frontoni M, Prencipe M, Fieschi C. A prospective study of cerebral ischemia in the young: analysis of pathogenic determinants. The National Research Council Study Group. Stroke 1993;24:362-367.
12. Bogousslavsky J, Van Melle G, Despland PA, Regli F. Alcohol consumption and carotid atherosclerosis in the Lausanne stroke registry. Stroke 1990;21:715-720.

13. Palomäki H, Kaste M, Raininko R, Salonen O, Juvela S, Sarna S. Risk factors for cervical atherosclerosis in patients with transient ischemic attack or minor ischemic stroke. Stroke 1993;24:970-975.

14. Silva JM, Mendez MM, Gonzalo M, Pinto E. J-curve between alcohol consumption and carotid atherosclerosis in patients with dyslipidemia. Ann Med Intern 2000;17:582-587.

15. Mukamal KJ, Kronmal RA, Mittleman MA, et al. Alcohol consumption and carotid atherosclerosis in older adults. The Cardiovascular Health Study. Arterioscler Thromb Vasc Biol, 2003;23:2252-2259.

16. Camargo Jr. CA Moderate alcohol consumption and stroke: the epidemiologic evidence. Stroke 1989;20:1611-1626.

17. Bo P, Marchioni E, Bosone D, et al. Effects of moderate and high doses of alcohol on carotid atherogenesis. Eur Neurol 2001;45:97-103.

18. Salonen JT, Seppänen K, Raurama AR, Salonen R. Risk for carotid atherosclerosis: the Kuopio Ischaemic Heart Disease Risk Factor Study. Ann Med 1989;21:227-229.

19. Djousse L, Myers RH, Province MA, et al. Influence of apolipoprotein E, smoking, and alcohol intake on carotid atherosclerosis: National Heart, Lung, and Blood Institute Family Heart Study. Stroke 2002; 33:1357-1361.

20. Barboriak JJ, Anderson AJ, Rimm AA, Tristani FE. Alcohol and coronary arteries. Alcohol Clin Exp Res,1979;3:29-32.

21. Dyer AR, Stamler J, Paul O, et al. Alcohol consumption, cardiovascular risk factors and mortality in two Chicago epidemiologic studies. Circulation 1977;56:1067-1074.

22. Klatsky AL, Friedman GD, Siegelaub AB. Alcohol consumption before myocardial infarction: results from the kaiser-permanent epidemiologic study of myocardial infarction. Ann Intern Med,1974;81:294-301.

23. Hillbom M, Kaste M, Rasi V. Can ethanol intoxication affect hemocoagulation to increase the risk of brain infarction in young adults? Neurology 1983;33:381-384.

24. Rosengren A, Wilhelmsen L, Pennert K, Berglund G, Elmfeldt D. Alcoholic intemperance, coronary heart disease and mortality in middleaged swedish men. Acta Med Scand,1987;222:201-213.

25. Kelbaek H, Heslet L,Skagen K, Christensen NJ, Godtfredsen J, Munck $\mathrm{O}$. Hemodynamic effects of alcohol at rest and during upright exercise in coronary artery disease. Am. J. Cardiol 1988;61:61-64.

26. Davidson D. Cardiovascular effects of alcohol. West J Med 1989;151:430439.

27. Puddey IB, Beilin LJ, Vandogen R, Rouse IL, Rogers P. Evidence for a direct effect of alcohol consumption on blood pressure in normotensive men - a randomized controlled trial. Hypertension 1985;7:707-713.

28. Ramos SP, Bertolote JM. Alcoolismo hoje. 2.Ed. Porto Alegre Artes Médicas, 1990.

29. Catapano AL. Antioxidant effect of flavonoids. Angiology 1997;48:39-44. 